\title{
Clinically useful screen for borderline personality disorder in psychiatric out-patients
}

\author{
Mark Zimmerman, Matthew D. Multach, Kristy Dalrymple and Iwona Chelminski
}

\begin{abstract}
Summary
A total of 3674 psychiatric out-patients were evaluated with a semi-structured diagnostic interview for DSM-IV borderline personality disorder (BPD). The affective instability criterion had a sensitivity of $92.8 \%$, higher than the sensitivities of the other eight BPD criteria. The negative predictive value of the affective instability criterion was $99 \%$. We recommend that clinicians screen for BPD in the same way that they screen for other psychiatric disorders: by enquiring about a single feature of the disorder (i.e. affective instability), the presence
\end{abstract}

of which identifies most patients with the disorder and the absence of which rules out the disorder.

\section{Declaration of interest}

None.

\section{Copyright and usage}

(c) The Royal College of Psychiatrists 2017.
Borderline personality disorder (BPD) is a serious illness resulting in high levels of psychosocial morbidity, ${ }^{1}$ reduced health-related quality of life, ${ }^{2}$ high use of services ${ }^{3}$ and excess mortality. ${ }^{4}$ The underrecognition of BPD has been identified as a significant clinical problem. ${ }^{5}$ When clinicians conduct a diagnostic interview they typically screen for disorders that are comorbid with the principal diagnosis by asking about the comorbid disorders' necessary feature or 'gate criterion'. For example, in a patient with a principal diagnosis of major depressive disorder the clinician would enquire about the presence of panic attacks, excessive worry or substance use to screen for the presence of panic disorder, generalised anxiety disorder (GAD) or a substance use disorder. In contrast, for polythetically defined disorders such as BPD there is no gate criterion because it is diagnosed on the basis of the presence of at least five of nine criteria, none of which is required to be present. The goal of the present report from the Rhode Island Methods to Improve Diagnostic Assessment and Services (MIDAS) project was to determine whether it is possible to identify one or two BPD criteria that could serve as 'gate' criteria to screen for the disorder. We hypothesised that affective instability, considered by Linehan to be of central importance to the clinical manifestations of BPD, ${ }^{6}$ could function as such a gate criterion because prior small-scale studies have found that it is the most frequent of the BPD criteria. $^{7-9}$

\section{Method}

The Rhode Island MIDAS project represents an integration of research methodology into a community-based out-patient practice affiliated with an academic medical centre. A comprehensive diagnostic evaluation is conducted upon presentation for treatment. In total, 3674 patients were interviewed by a diagnostic rater who administered a modified version of the Structured Clinical Interview for DSM-IV (SCID) ${ }^{10}$ and the BPD section of the Structured Interview for DSM-IV Personality (SIDP-IV). ${ }^{11}$ The majority of the patients were female $(60.2 \%)$, and White $(87.1 \%)$, with a mean age of 38.8 years $($ s.d.=3.4). The most common current diagnoses were major depressive disorder $(41.8 \%)$, panic disorder $(17.1 \%)$, social anxiety disorder $(28.1 \%)$ and GAD (21.9\%). By contrast, the prevalence of current alcohol use $(8.8 \%)$, drug use $(5.4 \%)$ and eating disorders $(0.6 \%)$ was low. As described elsewhere, the diagnostic raters were highly trained and monitored throughout the project to minimise rater drift. ${ }^{12}$ The reliability of assessing the individual BPD criteria was examined in 47 patients. The mean of the kappa coefficients of agreement was 0.71 , ranging from $\kappa=0.56$ (recurrent suicidal behaviour or self-injury) to $\kappa=0.83$ (stress-related paranoia or dissociation). The reliability of the affective instability criterion was 0.79 .

\section{Statistical analysis}

We computed the sensitivity, specificity, positive and negative predictive values of each of the nine BPD criteria. A symptom or diagnostic criterion functions well as a screen if almost all patients with the disorder have the symptom (i.e. high sensitivity), and almost all patients who screen negative do not have the disorder (i.e. high negative predictive value). We split the sample in half based on whether the patient had an odd or even identification number and examined the performance of each BPD criterion in both halves. Because the MIDAS project has been ongoing for approximately 20 years, during which time we have had more than 35 interviewers, we conducted a secondary analysis to determine whether the performance of the BPD screen remained consistent over time. For this analysis we divided the sample into thirds - the first 1225 patients who were evaluated, the next 1225 and the last 1224 patients.

\section{Results}

The prevalence of BPD was $10.6 \%(n=390)$ in the total sample. In both the odd- and even numbered subsamples the sensitivity of the affective instability criterion was greater than $90 \%$ (Table 1 ). The sensitivity of the affective instability criterion was consistently high throughout the course of the study (Table 1). The negative predictive value of the affective instability criterion was $99 \%$ in both subsamples and consistently high throughout the duration of the study. The affective instability criterion had the highest sensitivity in both men (94.5\%) and women (92.1\%) and the criterion with the next highest sensitivity was anger $(83.4 \%$ and $85.3 \%$ in the two subsamples). The sensitivity of a two-item screen of affective instability or anger was $97.4 \%$ and $97.5 \%$ in the two subsamples. There was thus a small increase in sensitivity over that of the affective instability criterion alone (97.4\% v. 92.8\%). 


\begin{tabular}{|c|c|c|c|c|}
\hline & Sensitivity, \% & Specificity, \% & $\begin{array}{c}\text { Positive predictive } \\
\text { value, } \%\end{array}$ & $\begin{array}{c}\text { Negative predictive } \\
\text { power, } \%\end{array}$ \\
\hline \multicolumn{5}{|l|}{ Odd-even split } \\
\hline Even numbered sample $(n=1837)$ & 94.3 & 81.6 & 37.5 & 99.2 \\
\hline Odd numbered sample $(n=1837)$ & 91.4 & 82.3 & 38.2 & 98.8 \\
\hline \multicolumn{5}{|l|}{ Temporal split } \\
\hline First third $(n=1225)$ & 92.5 & 76.9 & 32.8 & 98.8 \\
\hline Middle third $(n=1225)$ & 91.5 & 83.7 & 39.7 & 98.8 \\
\hline Last third $(n=1224)$ & 94.5 & 85.1 & 42.6 & 99.3 \\
\hline All patients $(n=3674)$ & 92.8 & 81.9 & 37.9 & 99.0 \\
\hline
\end{tabular}

\section{Discussion}

Our results indicated that asking about affective instability functions well as a clinically useful screen for BPD. More than $90 \%$ of the patients with BPD endorsed this criterion, and this finding remained consistent through the two decades of the MIDAS project during which time many different interviewers conducted the evaluations. Because the sensitivity of the affective instability criterion was, itself, above $90 \%$, the addition of another item only modestly enhanced its screening performance. The identification of a single BPD criterion that is present in the vast majority of patients diagnosed with BPD allows clinicians to follow their usual clinical practice when conducting a psychiatric review of systems - enquiring about the gate criteria of various disorders. The results of the present study are consistent with the results of other, smaller, studies that found that more than $90 \%$ of patients with BPD report affective instability, ${ }^{7-9}$ and it was the most frequent BPD criterion.

It is important to appreciate the distinction between screening and case identification. We are not suggesting that the diagnosis of BPD can be abbreviated to an assessment of the presence or absence of affective instability. The positive predictive value of this criterion was only $38 \%$; thus, the majority of patients who reported affective instability did not receive the diagnosis of BPD. Such is to be expected when screening for a disorder - most individuals who screen positive will not have the disorder when the more definitive diagnostic evaluation is conducted. Although the positive predictive value was only $38 \%$, the negative predictive value of the affective instability criterion was $99 \%$. Other studies have likewise found a negative predictive value above $95 \% .^{7-9}$ A clinician can therefore be highly confident in ruling out a diagnosis of BPD in patients who do not report affective instability.

Although the generalisability of any single-site study is limited a strength of the study was that the patients were unselected with regard to meeting any inclusion or exclusion criteria. Further confidence in the validity of the results comes from their consistency with other studies of the diagnostic efficiency statistics of the BPD criteria, which found that the affective instability criterion had a sensitivity above $90 \%$. Nonetheless, the results should be replicated in other psychiatric settings with a different mix of psychiatric diagnoses.

In conclusion, we recommend that clinicians screen for BPD in the same way that they screen for other psychiatric disorders: by asking about the single feature of the disorder that is present in most patients with the disorder and the absence of which effectively rules out the disorder. The diagnostic efficiency statistics of the affective instability criterion of BPD suggest that it can function as such a screening criterion and thus it should be added to a psychiatric review of systems.

Mark Zimmerman, MD, Matthew D. Multach, BA, Kristy Dalrymple, PhD, Iwona Chelminski, PhD, Department of Psychiatry and Human Behavior, Brown Medical School, Providence, Rhode Island, USA

Correspondence: Mark Zimmerman, MD, 146 West River Street, Providence, RI 02904, USA. Email: mzimmerman@lifespan.org

First received 27 Jan 2016, final revision 13 Apr 2016, accepted 17 Apr 2016

\section{References}

1 Skodol AE, Gunderson JG, McGlashan TH, Dyck IR, Stout RL, Bender DS, et al. Functional impairment in patients with schizotypal, borderline, avoidant, or obsessive-compulsive personality disorder. Am J Psychiatry 2002; 159: 276-83

2 Grant BF, Chou SP, Goldstein RB, Huang B, Stinson FS, Saha TD, et al. Prevalence, correlates, disability, and comorbidity of DSM-IV borderline personality disorder: results from the Wave 2 National Epidemiologic Survey on Alcohol and Related Conditions. J Clin Psychiatry 2008; 69: 533-45.

3 Bender DS, Dolan RT, Skodol AE, Sanislow CA, Dyck IR, McGlashan TH, et al. Treatment utilization by patients with personality disorders. Am J Psychiatry 2001; 158: 295-302.

4 Pompili M, Girardi P, Ruberto A, Tatarelli R. Suicide in borderline personality disorder: a meta-analysis. Nord J Psychiatry 2005; 59: 319-24.

5 Zimmerman M, Mattia Jl. Differences between clinical and research practice in diagnosing borderline personality disorder. Am J Psychiatry 1999; 156 1570-4

6 Linehan MM. Cognitive-Behavioral Treatment of Borderline Personality Disorder. Guilford Press, 1993.

7 Grilo CM, Becker DF, Anez LM, McGlashan TH. Diagnostic efficiency of DSM-IV criteria for borderline personality disorder: an evaluation in Hispanic men and women with substance use disorders. J Consult Clin Psychol 2004; 72: $126-31$

8 Pfohl B, Coryell W, Zimmerman M, Stangl D. DSM-III personality disorders: diagnostic overlap and internal consistency of individual DSM-III criteria. Compr Psychiatry 1986; 27: 22-34.

9 Nurnberg H, Raskin M, Levine P, Pollack S, Siegel O, Prince R. Hierarchy of DSM-III-R criteria efficiency for the diagnosis of borderline personality disorder. J Pers Disord 1991; 5: 211-24.

10 First MB, Spitzer RL, Gibbon M, Williams JBW. Structured Clinical Interview for DSM-IV Axis I Disorders - Patient edition (SCID-I/P, version 2.0). Biometrics Research Department, New York State Psychiatric Institute, 1995.

11 Pfohl B, Blum N, Zimmerman M. Structured Interview for DSM-IV Personality. American Psychiatric Press, 1997.

12 Zimmerman, M, Rothschild, L, Chelminski, I. The prevalence of DSM-IV personality disorders in psychiatric outpatients. Am J Psychiatry 2005; 162: $1911-8$. 